\title{
Interleukin-13 alters tight junction proteins expression thereby compromising barrier function and dampens rhinovirus induced immune responses in nasal epithelium
}

\author{
Zhi-Qun Huang ${ }^{1}$, Jing Liu ${ }^{2}$, Hsiao Hui Ong ${ }^{3}$, Tian Yuan ${ }^{4}$, Xiang-Min Zhou ${ }^{5}$, Jun Wang ${ }^{1}$, \\ Tan Kai-Sen ${ }^{6}$, Vincent Chow $^{3}$, Qin-Tai Yang ${ }^{7}$, Li Shi $^{5}$, Jing $\mathrm{Ye}^{1}$, and De Yun Wang ${ }^{8}$ \\ ${ }^{1}$ First Affiliated Hospital of Nanchang University \\ ${ }^{2}$ National University of Singapore - Kent Ridge Campus \\ ${ }^{3}$ National University Singapore Yong Loo Lin School of Medicine \\ ${ }^{4}$ The Third Affiliated Hospital Sun Yat-sen University \\ ${ }^{5}$ Second Hospital of Shandong University \\ ${ }^{6}$ National University of Singapore \\ ${ }^{7}$ The Third Affiliated Hospital, Sun Yat-sen University \\ ${ }^{8}$ Yong Loo Lin School of Medicine, National University of Singapore
}

June 4, 2020

\begin{abstract}
Background: Tight junctions (TJs) are intracellular structures which are essential for epithelial barrier function and play an important role in antimicrobial defense. Epithelium dysfunction and type-2-skewed inflammation are two main pathological phenomena of chronic rhinosinusitis with nasal polyps (CRSwNP). However, the effect of pro-inflammatory type-2 cytokine interleukin-13 (IL-13) on TJs in CRSwNP is poorly understood. Methods: Nasal biopsies of 70 CRSwNP patients and 25 healthy subjects, and in vitro IL-13-matured human nasal epithelial cells (hNECs) in 9 persons were used to analyze epithelial markers and TJ proteins. Epithelium permeability, transepithelial electrical resistance (TEER), mRNA and protein expression of TJs were quantified for IL-13-matured hNECs and that with RV infection. Results: Both mRNA and protein expression of occludin, claudin-3 and ZO-1 were significantly decreased in CRSwNP biopsies and in hNECs after IL-13 treatment. Differentiation of hNECs with IL-13 treatment increased epithelium permeability, decreased TEER and altered hNECs composition resulting in lesser ciliated cells and mucus over-secretion. Interestingly, claudin-3 is selectively expressed on ciliated cells. While RV infection induced minimal changes to TJs, the IL-13-matured hNECs has reduced capacity for upregulation of IFN- $\lambda 1$ and CXCL10 but further increased the expression of TSLP upon acute RV infection. Conclusions: These findings suggested that IL-13-mediated dysfunction of TJs and compromised epithelial barrier. IL-13-induced cilia loss conferred lowered viral replication and impaired antiviral responses of nasal epithelium against acute RV infection.
\end{abstract}

\section{Introduction}

The normal sinonasal epithelium comprises of ciliated cell, goblet cell, club cell and basal cell ${ }^{1}$. Tight junctions (TJs), which is located at the most apical part of intercellular junction between these epithelial cells, serves as a physical barrier of nasal airway epithelium to protect it from the external environment ${ }^{2}$. The main components of TJs are occludin, claudins, junctional adhesion molecules and scaffold protein zonula occludens (ZO). These TJ proteins are the key structural proteins which maintain epithelial polarity, regulate pericellular permeability and participate in epithelial cell proliferation, differentiation and migration ${ }^{3,4}$. These functions play an important role in distinct tissue compartmentalization and homeostasis of nasal 
epithelium. Disruption of TJs may cause reduction in epithelial cohesion and integrity which may lead to a variety of pathological conditions. It has been demonstrated that TJ proteins are involved in the pathophysiology of chronic airway inflammatory disorders including chronic rhinosinusitis with nasal polyps (CRSwNP), allergic rhinitis and asthma ${ }^{5-7}$.

IL-13 is the key regulator in type-2 mediated inflammation in CRSwNP. IL-13 induces goblet cell hyperplasia, loss of cilia, inducible nitric oxide syntheses production and fibrosis to accelerate inflammation and promote remodeling 8,9 . The elevated levels of pro-inflammatory cytokines contributing to diseases pathophysiology and barrier dysfunctions have been implicated in a variety of tissues ${ }^{9-11}$. Studies have shown that IL13 impaired barrier function by reducing expression of occludin, ZO-1 and $\beta$-catenin in primary bronchial epithelial cells of asthmatic patients ${ }^{12}$ and also disrupted intestinal barrier by upregulation of claudin$2^{13}$. Additionally, it was found that with IL-4/IL-13 and IL-5 stimulation, E-cadherin and ZO-1 were downregulated in cultured epithelial cells of patients with allergic rhinitis ${ }^{14}$. Hence, these studies further support the hypothesis that change in expression and localization of TJs accounts for epithelial barrier dysfunctions in chronic inflammatory diseases.

In CRSwNP, persistent and prolonged mucosal inflammation is well characterized and is closely related to elevated type-2 cytokines. However, the role of these cytokines on impairing the nasal epithelial barrier is still unknown. On the other hand, human nasal epithelial cells (hNECs), being the primary entry point of most inhaled pathogens, are the key players in regulating inflammatory responses and are an important source of pro-inflammatory cytokines ${ }^{15}$. Among respiratory viruses, rhinovirus (RV) is most commonly associated with exacerbation of chronic airway disease ${ }^{16,17}$. It was found that RV infection damaged TJs integrity in airway epithelial cells of asthmatic patients by reducing ZO-1, occludin and claudin-1 protein expression ${ }^{7}$. In addition, while it is reported that RV caused transient barrier disruption in a model of normal air-liquid interface (ALI) differentiated airway epithelium, RV infection at initial stage of differentiation in an injury model prolonged barrier dysfunction by decreasing transepithelial electrical resistance (TEER) and occludin level ${ }^{18}$. Furthermore, recent study has shown that RV infection increased production of pro-inflammatory mediators and contributed to the exaggerated inflammatory response in in vitro cancer cell line ${ }^{19}$. While RV is reported highly prevalent in chronic rhinosinusitis patients ${ }^{20}$, the underlying mechanism of the association of airway disease with chronic inflammation and virus comorbidity is still poorly understood.

Our previously established in vitro IL-13-matured hNECs model using IL-13 stimulation closely mimicked the physiological condition and epithelium responses of in vivo nasal mucosa in $\mathrm{CRSwNP}^{21}$. Using this model, we investigate the direct effect of IL-13 on hNECs and TJ proteins expression in pseudostratified layers to analyze the nasal epithelial barrier functions. Moreover, to better define the effects of respiratory viruses on TJs of inflammatory airway model, we have also analyzed the nasal epithelial barrier integrity, remodeling and immune responses of IL-13-treated hNECs against RV acute infection.

\section{Materials and methods}

\section{Study patients}

Nasal biopsy specimens were recruited from the Department of Otolaryngology, First Affiliated Hospital of Nanchang University; the Department of Otolaryngology, National University Hospital of Singapore; and the Department of Otolaryngology, Second Hospital of Shandong University. 70 CRSwNP patients who underwent functional endoscopic sinus surgery were recruited. CRSwNP diagnoses were made according to the current European position paper on rhinosinusitis and nasal polyps (EPOS 2020) ${ }^{22} .25$ control subjects did not have a history of sinonasal inflammation and inferior turbinate (IT) tissues were taken from patients who underwent septal plastic surgery. The ethics approval was obtained from the institutional review boards of the participating hospitals in China (2019124) and National Healthcare Group Domain Specific Review Board of Singapore (DSRB D/11/228 and IRB 13-509). All participants gave informed consent to participate in the study. The clinical characteristics of the study subjects were shown inTable 1 .

\section{Cell culture and IL-13 stimulation}


The hNECs were differentiated from human nasal epithelial stem/progenitor cells (hNESPCs) isolated from IT of healthy subjects $(n=9)$. The hNESPCs were transferred to an ALI system to form a pseudostratified layer within 4 weeks. Methods for culturing hNECs were described in previous paper ${ }^{23}$. IL-13 (10ng/ml, R\&D System, Minneapolis, MN, USA) was added in the medium on the first of ALI and medium with IL-13 were replenished every 2 days for 21 days until they were fully differentiated.

\section{Infection and quantification of RV}

Fully differentiated hNECs with and without IL-13 treatment were inoculated with RV16 (ATCC VR-283@) at a high dosage of MOI 10 and incubated at $33{ }^{\circ} \mathrm{C}$ for $1 \mathrm{~h}$. Apical wash and RV-infected and uninfected hNECs were harvested at $24 \mathrm{~h}$ post-infection. Plaque assay was performed as previously described ${ }^{24}$. The plaque-forming unit (PFU) was calculated as follows: Number of plaques $\times$ dilution factor $=$ number of PFU per $100 \mu l$.

\section{TEER}

The TEER of IL-13-matured hNECs was measured from Day3 to Day21 of ALI culture by using an EVOM voltammeter device with STX2 electrode (WPI, Sarasota, FL, USA). TEER was calculated by subtracting blank value. The total TEER $\left(\mathrm{ohms} \cdot \mathrm{cm}^{2}\right)$ is presented by TEER measurement (ohms) $\times$ Area of a membrane $\left(\mathrm{cm}^{2}\right)$.

\section{Barrier function assays}

The TJ permeability assay was performed according to previous study by using surface biotinylation ${ }^{25}$. Briefly, ALI-cultured hNECs were incubated with $1 \mathrm{mg} / \mathrm{ml}$ EZ-Link Sulfo-NHS-LC-biotin (557 Da; Thermo Fisher Scientific, Inc., Waltham, MA) in DPBS for $45 \mathrm{~min}$. After washing, the samples were fixed overnight with $4 \% \mathrm{PFA}$ at $4^{\circ} \mathrm{C}$, embedded in paraffin, and sectioned at a thickness of $4 \mu \mathrm{m}$. After antigen retrieval, sections were washed and blocked with goat serum, and then incubated for 30 min with streptavidin (Alexa Fluor 488 conjugate, Thermo Fisher Scientific).

\section{Immunofluorescence (IF) staining}

IF staining was performed for paraffin sections of nasal tissue, transwell membranes of ALI culture and cytospin clinical samples. Sample sections were blocked with $10 \%$ normal goat serum, then incubated with a primary antibody (Table E1 ) overnight at 4, followed by 60 min incubation of Alexa Fluor 488- or Alexa Fluor 594-conjugated secondary antibodies in the dark at room temperature. Mounting medium with 4'6-diamidino-2-phenylindole (DAPI) (Life Technologies, Carlsbad, CA) was used to stain cell nucleus.

\section{Total fluorescence intensity (TFI) evaluation}

Images of ZO-1, occludin, claudin-3 (Cldn3), MUC5AC and $\beta$ IV-tubulin on paraffin sections were captured at $400 \times$ magnification with a fluorescence microscope (Olympus IX51, Tokyo, Japan). Protein expression of these markers was analyzed using Image J software by calculating the value of positively stained area and the mean fluorescence intensity for each marker. TFI measurements were performed by multiplying the positive area by mean fluorescence intensity and corrected by subtracting the background autofluorescence.

\section{Immunoblotting}

The preparation of the cell lysates, SDS-PAGE and western blot analysis were performed according to standard protocols. Equal loading for each sample was used to detect ZO-1, occludin, Cldn3 and glyceraldehyde 3-phosphate dehydrogenase (GAPDH). The final assessment was evaluated as ratio of target protein to housekeeping protein.

\section{RNA isolation and quantitative real-time PCR}

Total RNA was extracted from frozen nasal tissues and hNECs using the mirVana miRNA Isolation Kit (Life Technologies, USA). Complementary DNA was synthesized in a $20 \mu \mathrm{l}$ reaction volume from $1 \mu \mathrm{g}$ total RNA using qScript cDNA SuperMix (Quanta BioDesign) according to the protocol of manufacturer. Relative 
genes expression was detected using SYBR green gene expression assays and was normalized to $2^{-\Delta^{a} \mathrm{~T}}$ with GAPDH and ribosomal protein L13a (RPL13A) as a housekeeping gene. The gene primer sequences used were shown inTable E2 .

\section{Statistical analysis}

All data were analyzed with GraphPad Prism 7 software (GraphPad Software, La Jolla, Calif). Fisher's exact test, Mann-Whitney U test and Wilcoxon signed-rank test were used to analyze differences between two groups. Two-way ANOVA was used to analyzed differences between hNECs with or without IL-13 treatment. Fold change was quantified with reference to untreated hNECs. Data were presented as Mean \pm SEM or Median with interquartile range (25\%-75\%). Correlation analysis was performed using Spearman $\mathrm{r}$ characteristic. $P<.05$ was considered statistically significant.

\section{Results}

\section{Aberrant expression and association of TJs and epithelial cell markers in nasal biopsy speci-} mens of CRSwNP patients.

First, we investigated the expression and localization of TJs proteins (ZO-1, occludin and Cldn3) and epithelial cell marker MUC5AC (goblet cell) and $\beta$ IV-tubulin (ciliated cell) in control subjects and CRSwNP patients. The epithelium in CRSwNP patients was damage as shown by loss of cilia and abnormal epithelium remodeling (goblet cell hyperplasia) by IF staining (figure $\mathbf{1 A - F}, \mathbf{G}-\mathbf{L}, \mathbf{P}$ and $\mathbf{Q}$ ). In control subjects, ZO-1 was consistently expressed and localized at superficial side of the nasal epithelium (figure 1A and G ) and occludin was localized at both superficially and suprabasally at the cell boundaries but a higher intensity at the superficial layer (figure $\mathbf{1 C}$ and $\mathbf{I}$ ). Cldn3 was found at the cell boundaries and at the superficial layer of nasal epithelium (figure 1E and $\mathbf{K}$ ). On the other hand, in CRSwNP patients, protein expression of ZO-1, occludin and Cldn3 was reduced (all $P<.001$; figure $\mathbf{1 B}, \mathbf{H}, \mathbf{M} ; \mathbf{D}, \mathbf{J}, \mathbf{N}$ and $\mathbf{F}, \mathbf{L}, \mathbf{O}$ ). Similarly, the mRNA expression levels of $Z O-1$, occludin and $C l d n 3$ were reduced in CRSwNP patients as compared to the control subject (figure 1R-T ).

Despite higher MUC5AC protein expression in CRSwNP, its mRNA expression was lower in tissue of CRSwNP $(P<.001$; figure 1U ) while there was no significant change in mRNA expression of Foxj1 (figure 1V ). Furthermore, we found that MUC5AC expression was negatively correlated with occludin $(r=-0.374, P$ $=.003$; figure 2B $)$ and $\mathrm{Cldn} 3(r=-0.386, P=.002$; figure 2C $)$ but not with $\mathrm{ZO}-1$ ( $r=-0.128, P=.328$; figure 2A ) in nasal biopsies. $\beta \mathrm{IV}$-tubulin expression was positively correlated with ZO-1 $(r=.529, P<.001$; figure 2D $)$, occludin $(r=.566, P<.001$; figure 2E $)$ and $\operatorname{Cldn} 3(r=.622, P<.001$; figure 2F $)$. Then, we examined the expression of TJs in ciliated and goblet cells by single cell staining of primary nasal cells. We found that both ZO-1 (figure $\mathbf{2 G}$ and $\mathbf{J}$ ) and occludin (figure $\mathbf{2} \mathbf{H}$ and $\mathbf{K}$ ) were co-stained with goblet and ciliated cell. Interestingly, Cldn3 staining was only observed in ciliated cell (figure 2L ) but not goblet cell (figure 2I ).

\section{IL-13 induces epithelial remodeling and disrupts epithelial barrier integrity in hNECs.}

To analyze the direct effect of IL-13 on nasal epithelial barrier functions, IL-13 was added throughout hNECs differentiation in ALI culture. Cilia loss and mucus over-secretion were observed in IL-13-matured hNECs (all $P=.016$; figure 3A-D ). Similar to the trend observed in nasal biospecimen, the protein expression of occludin and Cldn3 but not ZO-1 were significantly decreased in hNECs with IL-13 treatment (all $P=.016$; figure 3E-H ). IL-13 also disrupted ZO-1, occludin and Cldn3 as shown by an irregular staining patterns as compared to untreated hNECs (figure 3I-N ). In addition, epithelium permeability assay using SulfoNHS-Biotin as a tracer revealed that IL-13 increased the paracellular permeability of hNECs (figure 3O-P ). Long-term exposure of hNECs to IL-13 resulted in a reduction of TEER (figure $\mathbf{3 Q}$ ).

\section{IL-13 induced regulation of TJ genes during hNECs differentiation.}

Next, we analyzed the role of IL-13 on regulation of the TJs formation during hNECs differentiation. Cldn3 mRNA expression level was significantly reduced from Day11 onwards while the ZO-1 andoccludin mRNA 
expression were significantly reduced only when hNECs were fully differentiated at Day21(figure 4A-C ). IL13 upregulated and downregulated mRNA expression of MUC5AC andFoxj1 during hNECs differentiation respectively (figure $4 \mathrm{D}-\mathrm{E}$ ).

Positive IF staining of Cldn3 was detected when ciliated cells were first observed at Day11 and was colocalized with $\beta I V$-tubulin-positively stained cells during differentiation of untreated hNECs, while Cldn3 staining was only detected from Day15 onwards in IL-13-matured hNECs (figure 4F-G ). In addition, there was lower expression of Cldn3 observed in IL-13-matured hNECs with less ciliated cells as compared to more widespread expression pattern of Cldn3 in untreated controls with more ciliated cells. On the other hand, positive stained ZO-1 and occludin were observed to localize at cell-to-cell contact sites as early as Day7 and Day3 at early stage of differentiation of hNECs while their localizations were non-linear and fragmented at cell-to-cell boundaries in IL-13-matured hNECs (figure E1A-D ).

\section{IL-13 modulates the effects of RV infection in hNECs}

To better understand the effect of RV on TJs of inflammatory airway model, we next examine the nasal epithelial barrier integrity, remodeling (cilia and goblet cells) and immune responses of IL-13-treated hNECs against high dose of acute RV infection. Firstly, we found that RV progeny production and viral RNA expression were significantly increased with or without IL-13 treatment. Interestingly, RV progeny production and viral RNA expression were significantly lower in IL-13-treated hNECs as compared to RV-infected hNECs without IL-13 (all $P=.031$; figure 5A-B ).

RV infection upregulated mRNA levels of $Z O-1$ and occludinbut IF staining showed only slight decrease with or without IL-13 treatment (figure E2A-B and F-G ). However, RV infection showed reduced Cldn3 mRNA expression in both IL-13-treated and untreated hNECs, albeit not statistically significant $(P=.219$; figure 5C ). With IL-13 stimulation, RV infection further reduced Cldn3mRNA expression as compared to untreated group ( $P=.063$;figure 5C $)$. Similar to ZO-1 and occludin, IF staining showed that RV infection induced slight decrease in expression of Cldn3 in both IL-13-treated and untreated hNECs (figure 5M ).

We also investigated the effect of RV infection on the cell type of hNECs. RV infection upregulated the mRNA expression of MUC5AC in untreated hNECs but not in IL-13-treated hNECs as compared to their respective mock-infected controls (all $P=.031$; figure 5D ). Interestingly, RV infection downregulated Foxj1 mRNA levels in both untreated and IL-13-treated hNECs (all $P=.031$;figure $5 \mathbf{E}$ ). Representative images of IF staining showed that RV infection increased expression of MUC5AC and reduced $\beta I V$-tubulin expression in IL-13-treated hNECs as compared to uninfected IL-13-treated hNECs while RV infection induced slight changes of expression in untreated group (figure $5 \mathrm{~N}-\mathrm{O}$ ).

We also examine the innate immune responses of IL-13-treated hNECs against acute RV infection. The mRNA expression of RV receptor ICAM-1 was reduced in both IL-13-treated and untreated hNECs as compared to the respective mock-infected controls (all $P=.031$; figure $\mathbf{5 F}$ ). With IL-13 stimulation, RV infection further reduced ICAM-1 mRNA expression as compared to infected hNECs without IL-13 ( $P$ $=.094$;figure 5F ). Similarly, RV pathogen recognition receptor TLR3 was reduced in both IL-13-treated and untreated hNECs as compared to the respective uninfected controls (all $P=.031$;figure 5G ). Additionally, RV infection increased the mRNA expression of antiviral type III IFN (I $\Phi N-\lambda 1)$ and chemokine CXCL10 in both IL-13-treated and untreated hNECs (all $P=.031$;figure 5H-I ). However, IL-13-treated hNECs has reduced capacity for upregulation of antiviral responses against $\mathrm{RV}$ infection as compared to RV infection in untreated hNECs as showed by lower extent of mRNA upregulation for both $I \Phi N-\lambda 1$ and $C X C L 10(P=.063$ and .031; figure 5H-I ). Moreover, RV infection upregulated mRNA expression of $I L$ - 25 and $I L$-33 only in untreated hNECs but not in IL-13-treated group $(P=.031$; figure $\mathbf{5 J}-\mathbf{K})$, whereas TSLP mRNA was upregulated in both IL-13-treated and untreated groups. (all $P=.031$; figure 5L ). With IL-13 stimulation, RV infection further increased TSLP mRNA expression as compared to untreated group $(P=.063$; figure 5L ). Similar to $I L-25$ and $I L$-33, RV infection increased the mRNA expression of type-2 cytokine $I L-13$ in untreated but not IL-13-treated hNECs $(P=.031$; figure E2D $)$. There was no significant change in mRNA expression of $I L-5$ and $I L-17 A$ (figure E2C and $\mathbf{E}$ ) while $I L-4$ mRNA expression was undetectable (data 
not shown).

\section{Discussion}

Our study demonstrates an impaired epithelial barrier function in CRSwNP patients along with decreased protein and mRNA expression of ZO-1, occludin and Cldn3. Long-term exposure of IL-13 altered hNECs composition in conjunction with nasal epithelial barrier dysfunction and enhanced mucosal inflammation, as well as reduced capacity for antiviral response against acute RV infection. These observations extend the knowledge of dysregulation of TJs in chronic airway diseases such as CRSwNP. Most importantly, ALIcultured hNECs are shown to form functional barriers similar to that found in nasal biopsies. Using the IL-13-matured hNECs model, we have demonstrated that pro-inflammatory mediator IL-13 regulates the formation TJs and thereby disrupts the nasal epithelial barrier functions.

Nasal epithelial cells form a functional barrier which is mainly regulated by TJs. Recent studies have highlighted multiple defective TJs in patients with chronic airway diseases ${ }^{26}$ and reported that ZO-1, claudin1 , claudin- 4 and occludin contributed to the leaky barrier of airway epithelium ${ }^{14,27,28}$. In our study, we found that the expression of Cldn3, similar to ZO-1 and occludin, is significantly lower in sinonasal tissues of CRSwNP as compared to healthy controls. Moreover, we noticed that occludin and Cldn3 protein expression level negatively correlates with MUC5AC expression and positively correlates with $\beta I V$-tubulin expression in nasal biopsies specimens. It appears that alteration of nasal epithelial homeostasis during inflammation may impair TJs integrity. For this purpose, we used ALI-cultured hNECs with IL-13 stimulation which mimics the remodeling of nasal epithelium during chronic inflammation to explore the formation and disruption of TJs and thereby the IL-13-induced barrier damage.

We demonstrated that long-term exposure of hNECs to IL-13 results in overproduction of mucus and cilia loss along with impaired epithelial barrier function which is evident by increased epithelium permeability and decreased TEER. ZO-1, occludin and Cldn3 structures were also disrupted by IL-13 treatment during hNECs differentiation. In addition to enhanced inflammation, defective barrier function allows foreign substance to infiltrate the sinonasal submucosa and causes aggravation of airway inflammation and remodeling. Hence, TJs play an important in the pathophysiology of chronic airway disease. Taken together, these data indicate that impaired formation of TJs protein ZO-1, occludin and Cldn3 is largely associated with barrier dysfunction.

Although previous studies have examined TJs expression following various cytokine insults, differentiated epithelial cell lines or primary airway epithelial cells were commonly used ${ }^{14}$. However, these cell models were not able to elucidate the processes of TJs formation during epithelium differentiation. Therefore, we investigate the effect of IL-13 on TJs protein formation during the time course of ALI cultivation. We found that positively stained ZO-1 and occludin were observed as early as Day7 and Day3 respectively at early stage of differentiation of hNECs with or without IL-13 treatment. Interestingly, Cldn3 gene expression only increased when ciliated cells were first observed at Day11 and is expressed only on ciliated cells of hNECs. Hence, the reduced levels of Cldn3 may be attributed to loss of cilia in airway epithelium of chronic inflammatory airway disease. Additionally, as key barrier function proteins, claudins serve as paracellular barrier ${ }^{29}$ and are shown to have differential tissue-specific expression patterns which account for the differences in paracellular tightness and ion selectivity ${ }^{11,30,31}$. Although Cldn3 is expressed in the airways, its role in airway barrier function has not been fully defined. Studies in lower airways have demonstrated that the expression level and function of Cldn3 are not comparable between type-I and type-II alveolar cells ${ }^{32-34}$. Therefore, consistent with study which reported varying TJ composition in different airway epithelial cell type, the differential expression of Cldn3 in our study implies that Cldn3 may be involved in regulation of epithelial barrier as well as in epithelial differentiation. However, involvement Cldn3 in ciliogenesis during differentiation is currently unknown. Thus, further studies will be needed to investigate how TJ composition and epithelial cell differentiation interrelate for regulation of barrier permeability within stratified epithelia.

As the nasal airway is the primary target site for most respiratory viral infections, impaired epithelial barrier could lead to greater susceptibility against viral infection and dysregulation of host innate immune 
responses $^{35}$. RV is the most prevalent respiratory virus in CRSwNP patients and is the most commonly associated with exacerbation of chronic airway disease ${ }^{16,17,20}$. As RV infection may have significant implications in regulating the epithelial barrier function and mucosal inflammation of CRSwNP, we further investigate the effect of RV infection on TJs of IL-13-matured hNECs and the effects of IL-13 on the host responses of hNECs against RV infection. We found that the altered hNECs composition (cilia loss and mucus overproduction) in the presence of IL-13 is associated with the reduced RV replication (viral RNA level) and viral particle formation as compared to RV infection without IL-13 at the same initial infectious dose. As our previous study found that RV almost exclusively infected ciliated cells but not goblet and basal cells in in vitro $\mathrm{hNECs}^{24}$, cilia loss due to IL-13 may impede RV infection by reducing target cells for viral replication. However, despite lower viral replication and production, RV infection of IL-13-treated hNECs worsened the mucociliary function by further inducing loss of cilia as shown by reduction of Foxj1mRNA level and IF staining. While studies have reported that RV infection disrupted TJs in primary airway epithelial cells ${ }^{16,36}$, our data showed that RV infection induced minimal alteration to TJs proteins in hNECs with and without IL-13 treatment. In addition, we investigated the effects of IL-13 on innate immune responses of hNECs against RV infection. We found that viral entry receptor ICAM-1, RV-induced host pathogen sensor (TLR3) and antiviral immune responses (IFN- $\lambda 1$ and CXCL10) were upregulated in both untreated and IL-13-treated hNECs, suggesting that RV infection induced immune surveillance and antiviral responses even in inflammatory hNECs model. However, the capacity for interferon activation and chemokine signaling were impaired when hNECs is predisposed with IL-13 environment as shown by lower $I \Phi N-\lambda 1$ and $C X C L 10$ expression as compared untreated hNECs. In contrast, RV-induced greater upregulation of TSLP expression in IL-13-treated hNECs suggesting that RV infection in nasal epithelium predisposed with type-2 cytokine environment could lead to enhanced allergic inflammation which may further drive inflammation during RVinduced exacerbation of disease. Our current study investigated the effects of mild respiratory virus RV-16, which commonly associated to chronic respiratory disease exacerbations, on epithelium barrier function and immune capacity of hNECs in type-2 cytokine environment. While RV infection induced minimal change to TJs dysfunction, the impairment of efficient antiviral response in nasal epithelium may be attributed to IL-13-induced change in hNECs composition. More pathogenic respiratory viruses could be studied using this model to assess the epithelia barrier function and antiviral responses of chronic inflammatory airway disease.

In conclusion, IL-13, a typical type-2 cytokine, contributes to diminished barrier function and airway inflammation that are seen in CRSwNP patients. Knowledge about the dysregulation of TJs will help to better understand the pathophysiology of CRSwNP and define the specific mechanisms that link allergic inflammation and antiviral responses, which could lead to new strategies for the prevention and treatment of the disease.

\section{Author Contributions:}

D. Y. W and J. Y. designed the study; Z. Q. H. and J. L. wrote the manuscript and performed most of the experiments; H. H. O., K. S. T. and V. T. C. performed the experiments; T. Y., X. M. Z., J. W., Q. T. Y. and L. S. collected the clinical and hNECs samples.

\section{Conflict of Interest Statement:}

The authors declare no conflict of interest.

\section{References}

1. Heffler E, Landi M, Caruso C, et al. Nasal cytology: Methodology with application to clinical practice and research. Clin Exp Allergy.2018;48(9):1092-1106.

2. Holgate ST. Epithelium dysfunction in asthma. J Allergy Clin Immunol. 2007;120(6):1233-1244; quiz $1245-1236$.

3. Kojima T, Go M, Takano K, et al. Regulation of tight junctions in upper airway epithelium. Biomed Res Int. 2013;2013:947072. 
4. Zihni C, Balda MS, Matter K. Signalling at tight junctions during epithelial differentiation and microbial pathogenesis. J Cell Sci. 2014;127(16):3401-3413.

5. Tian T, Zi X, Peng Y, et al. H3N2 influenza virus infection enhances oncostatin M expression in human nasal epithelium. Exp Cell Res.2018;371(2):322-329.

6. Steelant B, Farre R, Wawrzyniak P, et al. Impaired barrier function in patients with house dust miteinduced allergic rhinitis is accompanied by decreased occludin and zonula occludens-1 expression. $J$ Allergy Clin Immunol. 2016;137(4):1043-1053 e1045.

7. Looi K, Buckley AG, Rigby PJ, et al. Effects of human rhinovirus on epithelial barrier integrity and function in children with asthma.Clin Exp Allergy. 2018;48(5):513-524.

8. Doran E, Cai F, Holweg CTJ, et al. Interleukin-13 in Asthma and Other Eosinophilic Disorders. Front Med (Lausanne). 2017;4:139.

9. Fulkerson PC, Fischetti CA, Hassman LM, Nikolaidis NM, Rothenberg ME. Persistent effects induced by IL-13 in the lung. Am J Respir Cell Mol Biol. 2006;35(3):337-346.

10. Saatian B, Rezaee F, Desando S, et al. Interleukin-4 and interleukin-13 cause barrier dysfunction in human airway epithelial cells. Tissue Barriers. 2013;1(2):e24333.

11. Bauer HC, Krizbai IA, Bauer H, Traweger A. "You Shall Not Pass"-tight junctions of the blood brain barrier. Front Neurosci.2014;8:392.

12. de Boer WI, Sharma HS, Baelemans SM, et al. Altered expression of epithelial junctional proteins in atopic asthma: possible role in inflammation. Can J Physiol Pharmacol. 2008;86(3):105-112.

13. Luettig J, Rosenthal R, Barmeyer C, et al. Claudin-2 as a mediator of leaky gut barrier during intestinal inflammation. Tissue Barriers. 2015;3(1-2):e977176.

14. Lee HJ, Kim B, Im NR, et al. Decreased expression of E-cadherin and ZO-1 in the nasal mucosa of patients with allergic rhinitis: Altered regulation of E-cadherin by IL-4, IL-5, and TNF-alpha. Am J Rhinol Allergy. 2016;30(3):173-178.

15. Loxham M, Davies DE, Blume C. Epithelial function and dysfunction in asthma. Clin Exp Allergy. 2014;44(11):1299-1313.

16. Sajjan U, Wang Q, Zhao Y, et al. Rhinovirus disrupts the barrier function of polarized airway epithelial cells. Am J Respir Crit Care Med. 2008;178(12):1271-1281.

17. Kennedy JL, Turner RB, Braciale T, et al. Pathogenesis of rhinovirus infection. Curr Opin Virol. 2012;2(3):287-293.

18. Faris AN, Ganesan S, Chattoraj A, et al. Rhinovirus Delays Cell Repolarization in a Model of Injured/Regenerating Human Airway Epithelium. Am J Respir Cell Mol Biol. 2016;55(4):487-499.

19. Herbert C, Do K, Chiu V, et al. Allergic environment enhances airway epithelial pro-inflammatory responses to rhinovirus infection. Clin Sci (Lond). 2017;131(6):499-509.

20. Cho GS, Moon BJ, Lee BJ, et al. High rates of detection of respiratory viruses in the nasal washes and mucosae of patients with chronic rhinosinusitis. J Clin Microbiol. 2013;51(3):979-984.

21. Liu J, Li YY, Andiappan AK, et al. Role of IL-13R $\alpha 2$ in modulating IL-13-induced MUC5AC and ciliary changes in healthy and CRSwNP mucosa.Allergy. 2018;73(8):1673-1685.

22. Fokkens WJ, Lund VJ, Hopkins C, et al. European Position Paper on Rhinosinusitis and Nasal Polyps 2020. Rhinology. 2020;58(Suppl S29):1-464.

23. Li YY, Li CW, Chao SS, et al. Impairment of cilia architecture and ciliogenesis in hyperplastic nasal epithelium from nasal polyps. J Allergy Clin Immunol. 2014;134(6):1282-1292. 
24. Tan KS, Ong HH, Yan Y, et al. In Vitro Model of Fully Differentiated Human Nasal Epithelial Cells Infected With Rhinovirus Reveals Epithelium-Initiated Immune Responses. J Infect Dis.2018;217(6):906915.

25. Chen YH, Merzdorf C, Paul DL, Goodenough DA. COOH Terminus of Occludin Is Required for Tight Junction Barrier Function in Early Xenopus Embryos. J Cell Biol. 1997;138(4):891-899.

26. Buckley A, Turner JR. Cell Biology of Tight Junction Barrier Regulation and Mucosal Disease. Cold Spring Harb Perspect Biol.2018;10(1).

27. Jacob AM, Gaver DP, 3rd. Atelectrauma disrupts pulmonary epithelial barrier integrity and alters the distribution of tight junction proteins ZO-1 and claudin 4. J Appl Physiol (1985). 2012;113(9):1377-1387.

28. Gan H, Wang G, Hao Q, et al. Protein kinase D promotes airway epithelial barrier dysfunction and permeability through down-regulation of claudin-1. J Biol Chem. 2013;288(52):37343-37354.

29. Gunzel D, Yu AS. Claudins and the modulation of tight junction permeability. Physiol Rev. 2013;93(2):525-569.

30. Coyne CB, Gambling TM, Boucher RC, et al. Role of claudin interactions in airway tight junctional permeability. Am J Physiol Lung Cell Mol Physiol. 2003;285(5):L1166-1178.

31. Markov AG, Aschenbach JR, Amasheh S. Claudin clusters as determinants of epithelial barrier function. IUBMB Life.2015;67(1):29-35.

32. Wang F, Daugherty B, Keise LL, et al. Heterogeneity of claudin expression by alveolar epithelial cells. Am J Respir Cell Mol Biol. 2003;29(1):62-70.

33. Yu AS, McCarthy KM, Francis SA, et al. Knockdown of occludin expression leads to diverse phenotypic alterations in epithelial cells.Am J Physiol Cell Physiol. 2005;288(6):C1231-1241.

34. Mitchell LA, Overgaard CE, Ward C, et al. Differential effects of claudin-3 and claudin-4 on alveolar epithelial barrier function. Am J Physiol Lung Cell Mol Physiol. 2011;301(1):L40-49.

35. Vareille M, Kieninger E, Edwards MR, et al. The airway epithelium: soldier in the fight against respiratory viruses. Clin Microbiol Rev. 2011;24(1):210-229.

36. Yeo NK, Jang YJ. Rhinovirus infection-induced alteration of tight junction and adherens junction components in human nasal epithelial cells. Laryngoscope. 2010;120(2):346-352.

Table 1. Characteristics of subjects providing sinus tissue.

\begin{tabular}{llll}
\hline & Healthy control subjects & Patients with CRSwNP & $P$ value $^{*}$ \\
\hline Sample size (No.) & 25 & 70 & NA. \\
Gender (M/F) & $16 / 9$ & $47 / 23$ & .969 \\
Age (y, Mean \pm SEM) & $35.84 \pm 3.52$ & $40.25 \pm 12.65$ & .337 \\
Atopy (N\%) & $3(12 \%)$ & $16(23 \%)$ & .355 \\
Asthma (N\%) & $0(0 \%)$ & $11(16 \%)$ & .081 \\
Smoker (N\%) & $2(8 \%)$ & $12(17 \%)$ & .390 \\
\hline
\end{tabular}

NA, not applicable; M, male; F, Female.

${ }^{*} P$ value was evaluated using the Fisher's exact test. $P<.05$ was considered statistically significant.

Table E1. Antibodies for staining. 


\begin{tabular}{|c|c|c|c|c|c|}
\hline$\overline{\text { Antibody }}$ & Type & Dilution IF WB & Dilution IF WB & Catalog number & Company \\
\hline$\overline{\text { Antibody }}$ & Type & Dilution IF WB & Dilution IF WB & Catalog number & Company \\
\hline $\mathrm{ZO}-1$ & $\mathrm{pAb}$ & $1 / 400$ & $1 / 1000$ & HPA001636 & Sigma \\
\hline ZO-1 & $\mathrm{mAb}$ & $1 / 400$ & & $33-9100$ & $\begin{array}{l}\text { Thermo Fisher } \\
\text { Scientific }\end{array}$ \\
\hline Occludin & $\mathrm{pAb}$ & $1 / 400$ & $1 / 1500$ & $71-1500$ & $\begin{array}{l}\text { Thermo Fisher } \\
\text { Scientific }\end{array}$ \\
\hline Cldn3 & $\mathrm{pAb}$ & $1 / 400$ & $1 / 1000$ & ab15102 & Abcam \\
\hline MUC5AC & pAb & $1 / 500$ & & sc-20118 & $\begin{array}{l}\text { Santa Cruz } \\
\text { Biotechnology }\end{array}$ \\
\hline MUC5AC & $\mathrm{mAb}$ & $1 / 500$ & & ab24071 & Abcam \\
\hline$\beta I V$-tubulin & $\mathrm{mAb}$ & $1 / 500$ & & ab11315 & Abcam \\
\hline GAPDH & $\mathrm{mAB}$ & & $1 / 10000$ & ab8245 & Abcam \\
\hline
\end{tabular}

IF: Immunofluorescence; WB: Western blotting; pAb: rabbit polyclonal antibody; mAb: mouse monoclonal antibody.

Table E2. Primer sequences used for RT-qPCR.

\begin{tabular}{lll}
\hline Gene & Forward primer & Reverse primer \\
\hline ZO-1 & TTGTCTTCAAAAACTCCCAC & GACTCACAGGAATAGCTTTAG \\
Occludin & GGACTGGATCAGGGAATATC & ATTCTTTATCCAAACGGGAG \\
Cldn3 & CTGCATGGACTGTGAAAC & AAAATATCAAGTGCCCCTTC \\
$M U C 5 A C$ & AATGGTGGAGATTTTGCAC & TTCTTGTTCAGGCAAATCAG \\
Foxj1 & GTGAAGCCTCCCTACTC & TTCTTGTTCAGGCAAATCAG \\
$R V-16$ & GCACTTCTGTTTCCCC & CGGACACCCAAAGTAG \\
$I C A M-1$ & ACCATCTACAGCTTTCG & TCACACTTCACTGTCACC \\
$T L R 3$ & AGATTCAAGGTACATCATGC & CAATTTATGACGAAAGGCAC \\
$I \Phi N-\lambda 1$ & CAGGTTCAAATCTCTGTCAC & AACTCCAGTTTTTCAGCTTG \\
$C X C L 10$ & AAAGCAGTTAGCAAGGAAAG & TCATTGGTCACCTTTAGTG \\
$I L-25$ & CAGGTGGTTGCATTCTTGGC & GAGCCGGTTCAAGTCTCTGT \\
$I L-33$ & GCTGGGAAATAAGGTGTAC & CCAGAAGTCTTTTGTAGGA \\
$T S L P$ & AAAGTACCGAGTTCAACAAC & GTAGCATTTATCTGAGTTTCCG \\
$I L-4$ & TCACATTGTCACTGCAAATC & CCTTCTCAGTTGTGTTCTTC \\
$I L-5$ & AACTGTGCACTGAAGAAAC & CTAGGAATTGGTTACTCTCC \\
$I L-13$ & ATCACCCAGAACCAGAAG & ATGCAAGCTGGAAAACTG \\
$I L-17 A$ & GTATGAGAAAAGTCAGCCC & TGGTTACGATGTGAAACTTG \\
$G A P D H$ & ACAGTTGCCATGTAGACC & TTGAGCACAGGGTACTTTA \\
$R P L 13 A$ & GTCTGAAGCCTACAAGAAAG & TGTCAATTTTCTTCTCCACG \\
\hline
\end{tabular}

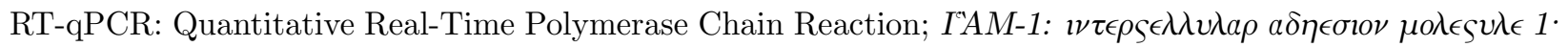

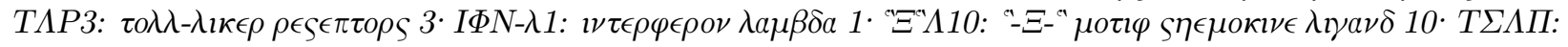

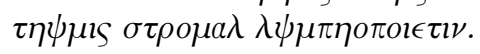

\section{Figure legends}

Figure 1.Aberrant expression of TJs and epithelial cell markers in nasal biopsy specimens of 
CRSwNP patients. Representative IF staining of ZO-1 (A-B, G-H ), occludin (C-D, I-J ), Cldn3 (E-F, K-L ) with MUC5AC (A-F ) and $\beta I V$-tubulin (G-L ) in nasal biopsies from control subjects and CRSwNP patients. TFI level of ZO-1, occludin, Cldn3 and $\beta I V$-tubulin were decreased in CRSwNP (M-Oand $\mathbf{Q}$ respectively) whereas MUC5AC TFI level was increased in CRSwNP patients (P ). Similarly, mRNA levels of occludinand Cldn3 were significantly decreased in CRSwNP as compared to control subjects(S-T ). There was no significant change in mRNA expression of $Z O-1$ and Foxj1 between two groups $(\mathbf{R}, \mathbf{V})$. Despite higher protein expression of MUC5AC as shown by IF staining in CRSwNP, its mRNA level was lower in CRSwNP tissue (U ). Relative expression of the target gene was normalized to $2^{-\Delta^{\prime \prime} T}$ with $G A P D H$. Statistical analysis was calculated using the Mann-Whitney U test. Data were presented as median with an interquartile range. Scale bar $=20 \mu \mathrm{m}$.

Figure 2. Correlation of TJs with goblet and ciliated cell in nasal biospecimen. TFI level of MUC5AC was not correlated with ZO-1 (A ) but was negatively correlated with occludin and Cldn3 (B-C ). $\beta$ IV-tubulin TFI level was positively correlated with ZO-1, occludin and Cldn3 (D-F ) in CRSwNP and control subjects. IF staining of single primary nasal cells showed that both ZO-1 ( G, J $)$ and occludin $(\mathbf{H}$, $\mathbf{K}$ ) were co-stained with goblet and ciliated cell. Interestingly, Cldn3 positive staining was only observed in ciliated cell (L), but not goblet cell (I ). Spearman $\mathrm{r}$ characteristic was performed for statistical analysis. Black dot refers to CRSwNP $(n=40)$ and red dot refers to control subjects $(n=20)$. Scale bar=10 $\mu$ m.

Figure 3. IL-13 induces epithelial remodeling and disrupts epithelial barrier integrity in hNECs. Representative IF images showed that IL-13 treatment induced more MUC5AC-secreting goblet cells and loss of ciliated cells in hNECs (A-D ). Western blotting results indicated that the protein expression of occludin and Cldn3 but not ZO-1 were significantly decreased with IL-13 treatment (E-H ). Damaged TJs proteins were shown by irregular staining patterns of ZO-1, occludin and Cldn3 in IL-13-matured hNECs as compared to untreated controls (I-N ). Intercellular epithelium permeability of IL-13-matured hNECs increased as compared to untreated hNECs (O-P ). IL-13 treatment of hNECs during differentiation also significantly reduced TEER at Day21 of ALI culture $(\mathbf{Q})$. Two-way ANOVA was used to analyze differences between hNECs with and without IL-13 treatment. Data were presented as median with an interquartile range. Fold change was quantified with reference to untreated hNECs. Scale bar $=20 \mu \mathrm{m}$. hNECs, $\mathrm{n}=7$.

Figure 4. Regulation of TJs protein expression during hNECs differentiation. IL-13 decreased the mRNA expression of ZO-1, occludin, Cldn3 and Foxj1 but increased MUC5AC mRNA level in IL-13matured hNECs as compared to untreated group (A-E ). IF staining was performed for Cldn3, MUC5AC, and $\beta I V$-tubulin from Day3 to Day21 during hNECs differentiation (F-G ). Cldn3 was only detected when ciliated cells were first observed at Day11 and Cldn3 was co-stained with $\beta I V$-tubulin during hNECs differentiation. Cldn3 localization is weakly detectable at poorly ciliated areas of hNECs induced by IL-13 whereas Cldn3 localization was more widespread in regions with more fully ciliated cells in untreated hNECs. The relative target gene was normalized to $2^{-\Delta^{a} T}$ with RPL13A as a housekeeping gene. Two-way ANOVA was used to analyzed differences between hNECs with and without IL-13 treatment. Data were presented as median with an interquartile range. Scale bar $=20 \mu \mathrm{m}$. hNECs, $n=9$.

Figure 5. Effects of RV infection on TJs and innate immune response on IL-13-treated hNECs. The RV progeny production and viral RNA expression were significantly increased for both untreated and IL13-treated hNECs. Extent of RV progeny production and viral RNA expression were significantly lower in IL13-treated hNECs as compared to untreated hNECs (A-B ). RV infection induced trend of reduction of Cldn3 mRNA expression in both IL-13-treated and untreated hNECs (C). RV infection only significantly increased the mRNA expression of MUC5AC in untreated but not in IL-13-treated hNECs. RV downregulated Foxj1 mRNA levels for both untreated and IL-13-treated hNECs. With IL-13 treatment, regulation of mRNA level of MUC5AC and Foxj1 were significantly higher and lower respectively than in untreated hNECs (D-E ). RV infection significantly increased the mRNA expression of RV receptor $I C A M-1$, pathogen recognition 
receptor TLR3 and antiviral $I \Phi N-\lambda 1$ and $C X C L 10$ in both untreated and IL-13-treated hNECs. With IL-13 treatment, upregulation of mRNA level of CXCL10 was significantly lower than in untreated hNECs (F-I ). RV infection significantly upregulated mRNA expression of $I L$-25 and $I L$-33 for untreated hNECs but not IL-13-treated hNECs (J-K ). RV significantly regulated the mRNA expression of TSLP in both untreated and IL-13-treated hNECs (L ). IF staining showed that RV infection induced slight decrease in expression of Cldn3 in IL-13-treated hNECs while expression of MUC5AC and $\beta I V$-tubulin was respectively higher and lower in IL-13-treated hNECs with RV infection (M-O ). The relative target gene was normalized to $2^{-\Delta^{\prime \prime T}}$ with RPL13A as a housekeeping gene. Two-tailed unpaired t-test was used to analyzed differences between hNECs with and without IL-13 treatment. Data were presented as median with an interquartile range. Fold change was quantified with reference to untreated hNECs. Scale bar $=20 \mu \mathrm{m}$. hNECs, $n=6$.

Figure E1. Expression of TJ proteins during hNECs differentiation. Positive staining of ZO-1 and occludin was observed at cell to cell contact sites even in early stage of differentiation. Localization pattern was not linear but fragmented at cell to cell boundaries after IL-13 treatment (A-D ). Scale bar=20 $\mu \mathrm{m}$.

Figure E2. Effects of RV infection on TJs and innate immune response on IL-13-treated hNECs. ZO-1 and occludinmRNA levels were increased in both untreated and IL-13-treated hNECs. RV significantly regulated $Z O-1$ and occludin mRNA levels in both untreated and IL-13-treated hNECs (A-B ). There was no significant change in mRNA expression of $I L-5$ (C) while $I L-4$ mRNA expression was undetectable (data not shown). RV infection significantly increased the mRNA expression of $I L-13$ in untreated but not IL-13-treated hNECs. RV significantly upregulated mRNA expression of $I L-13$ for untreated hNECs but not IL-13-treated hNECs (D ). There was no significant change in mRNA expression of $I L-17 A(\mathbf{E})$. Representative images of IF staining showed that RV infection in both untreated and IL13-treated hNECs induced slight increase in expression of ZO-1 and occludin (F-G ). The relative target gene was normalized to $2^{-\Delta^{a} \mathrm{~T}}$ with $R P L 13 A$ as a housekeeping gene. Two-tailed unpaired t-test was used to analyzed differences between hNECs with and without IL-13 treatment. Data were presented as median with an interquartile range. Fold change was quantified with reference to untreated hNECs. Scale bar=20 $\mu \mathrm{m}$. hNECs, $\mathrm{n}=6$.
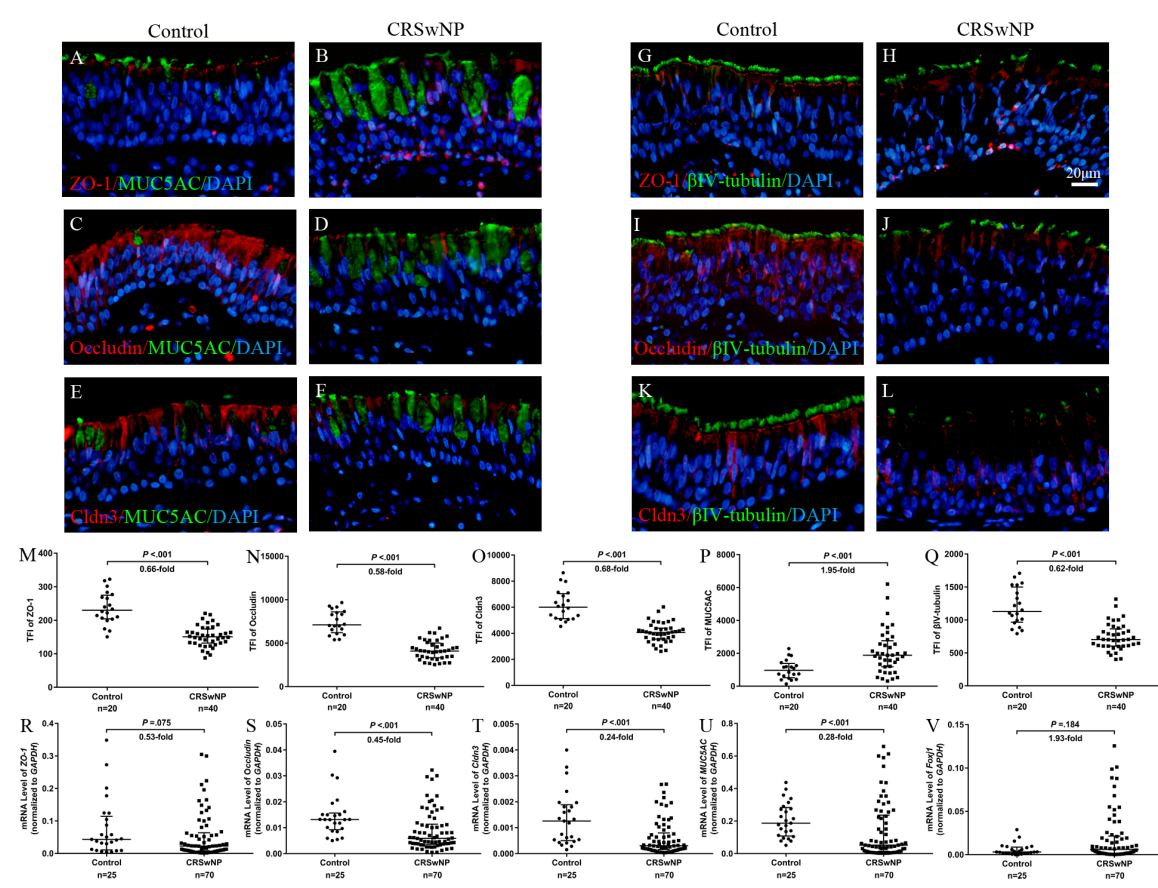

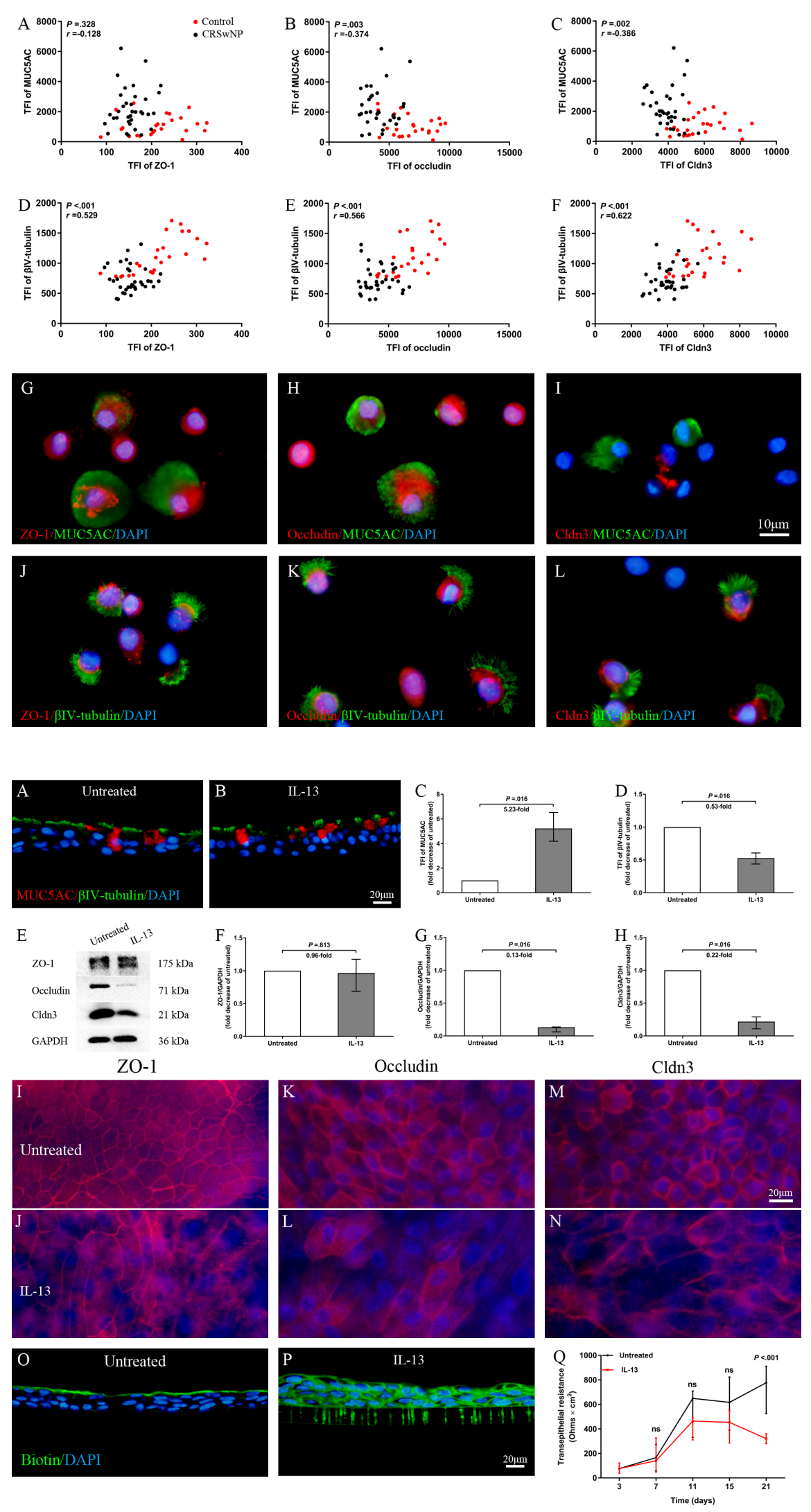

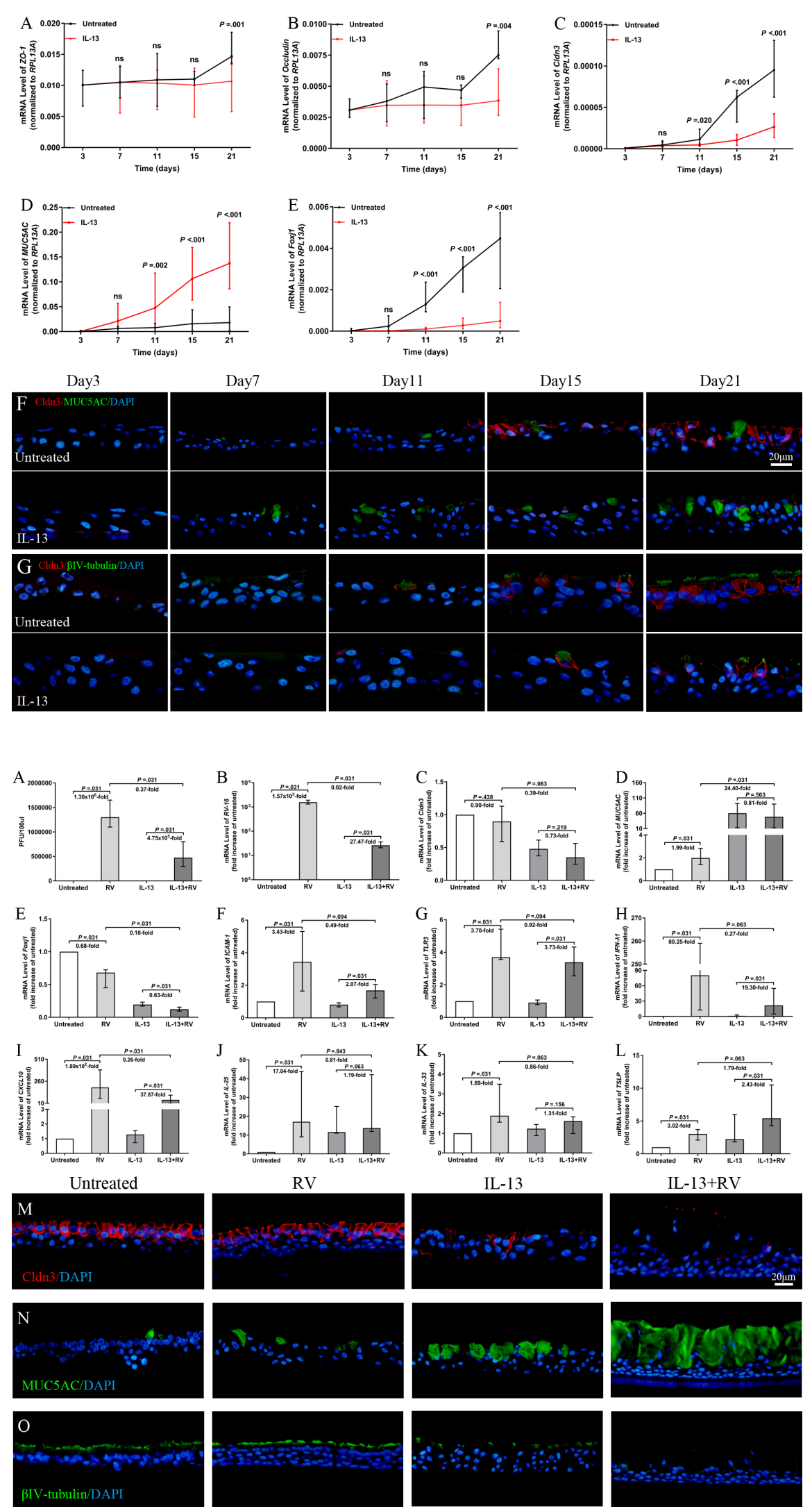

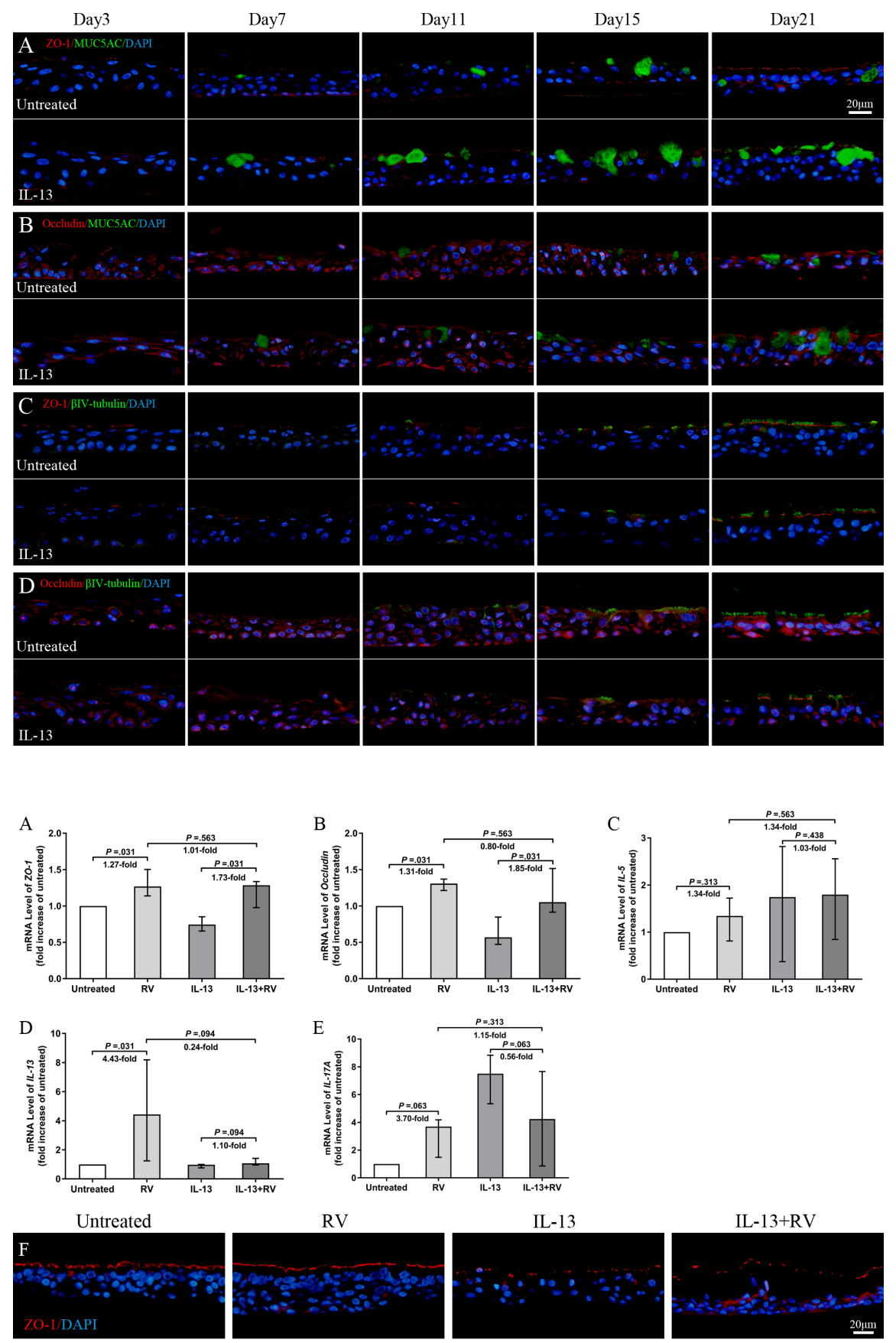